\title{
The Thawing Characteristic of Frozen Tofu under High-Voltage Alternating Electric Field
}

\author{
Shilong Deng, Zhiwei Gao, Jing Xu, Guoqing Wang, Yu Bai, and Changjiang Ding \\ Physical Experiment Center, College of Science, Inner Mongolia University of Technology, Hohhot, China \\ Correspondence should be addressed to Changjiang Ding; ding9713@163.com
}

Received 5 April 2017; Accepted 28 May 2017; Published 21 June 2017

Academic Editor: Srinivas Janaswamy

Copyright ( 2017 Shilong Deng et al. This is an open access article distributed under the Creative Commons Attribution License, which permits unrestricted use, distribution, and reproduction in any medium, provided the original work is properly cited.

\begin{abstract}
To systematically and comprehensively investigate the high voltage alternating electric field (HVAEF) thawing processing, we investigated the high-voltage electric field thawing characteristic of the frozen tofu at different voltages for alternating current (AC). The thawing time, thawing loss of frozen tofu, and specific energy consumption (SEC) of HVEF system were measured. Seven different mathematical models were then compared to simulate thawing time curves based on root mean square error, reduced mean square of deviation, and modeling efficiency. The results showed that the thawing rate of frozen tofu was notably greater in the high-voltage electric field system when compared to control. Both Linear and Quadratic models were the best mathematical models. Therefore, this work presents a facile and effective strategy for experimentally and theoretically determining the HVAEF thawing properties of frozen tofu.
\end{abstract}

\section{Introduction}

Tofu is representative of Chinese traditional food and is a favorite of consumers because of its unique flavor, rich taste, edible convenience, and rich nutritional value. Thawing of frozen materials is an important component of food processing, while freezing is a well-established process for food preservation and is confirmed to increase the storage time significantly. At present, the conventional thawing methods of frozen tofu include cold and warm water thawing, still air thawing, and refrigerator thawing. However, many disadvantages accompany such methods, including higher color deterioration and weight loss, longer thawing time, and decreased nutritional value. Consequently, growing interest has been shown in exploring new thawing methods for frozen tofu.

High-voltage electric field (HVEF) thawing is a relatively new, nonthermal technique [1], and it has been investigated by many researchers in pork [2,3], tuna fish [4-6], chicken [7], and apple tissue [8]. The advantages typically include reduced thawing time, food quality preservation, microbial growth inhibition, and reduced energy consumption. When the thawing temperature was set at $-3^{\circ} \mathrm{C}$, the thawing time of frozen chicken under high-voltage electric field was $2 / 3$ the time taken for thawing meat using a common refrigerator [7].
The HVEF treatment significantly shortened the thawing time of frozen pork tenderloin meat, and thawing time was reduced to $2 / 3$ that of the control [2]. Similarly, in the case of tune fish, HVEF significantly improved the thawing rate [4]. He et al. [9] reported that the thawing time can be maximally reduced by $50 \%$ compared to the conventional air thawing treatment. HVEF treatment reduced the total microbial counts in thawed frozen meat by $0.5-1 \log \mathrm{CFU} / \mathrm{g}$, without affecting meat quality, and reduced volatile basic nitrogen production during storage [2]. Parameters such as voltage, distance, and electric field strength were studied to determine the relationships and factors that affect HVEF treatment $[3,5]$. Mousakhani-Ganjeh et al. reported that the high-voltage electric field could increase susceptibility of tuna fish to lipid oxidation due to ozone generation [6]. The energy consumption of HVEF thawing was comparatively far smaller compared to the other thawing methods $[3,5]$. These studies were performed under high-voltage direct current electric field. To the best of our knowledge, few studies have systematically and comprehensively reported on the use of highvoltage alternating electric field (HVAEF) for thawing tofu.

To further investigate the potential of this method for optimizing and improving the thawing efficiency, frozen tofu 


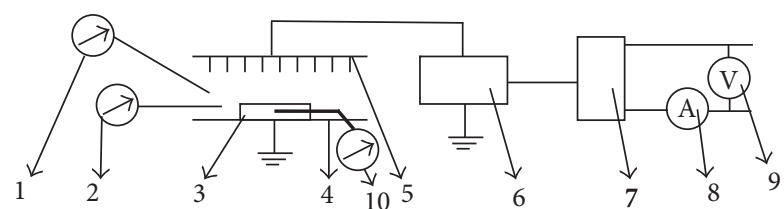

(a)

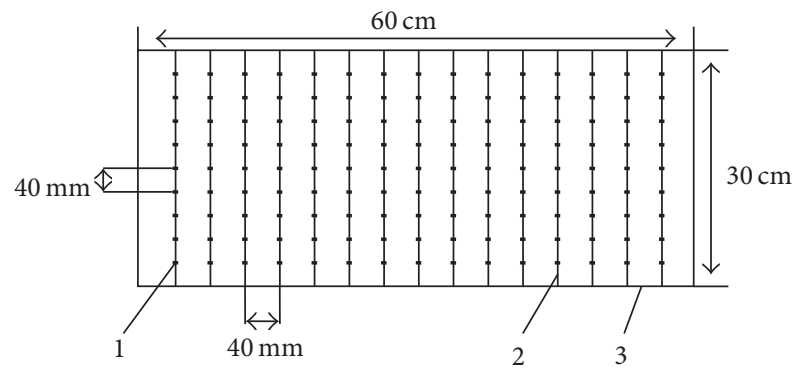

(b)

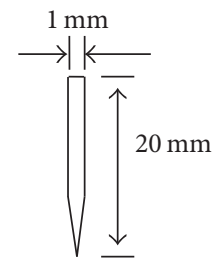

(c)

FIGURE 1: (a) Schematic diagram of HVAEF thawing: 1: thermometer; 2: hygrometer; 3 : frozen tofu; 4: grounded plate electrode; 5: needle electrodes; 6 : high-voltage power source; 7: voltage regulator; 8: ampere meter; 9: voltmeter; 10: temperature sensor. (b) Arrangement diagram of needle electrodes: 1: needle electrodes; 2: stainless steel wire; 3: stainless steel frame. (c) Schematic diagram of needle electrode.

was thawed in order to study the effect of voltage on thawing rate. The center temperature was measured, in addition to thawing loss and specific energy consumption of frozen tofu to understand the roles of energy consumption and product quality on thawing process.

\section{Materials and Methods}

2.1. Experimental Equipment. The lab-scale experimental setup for HVAEF thawing is shown schematically in Figure 1(a). This setup is similar to the EHD drying system [10]. It consists of a vertically mounted electrode with multiple sharp pointed needles projected to a fixed horizontal grounded metallic plate on which the frozen tofu samples to be thawed were placed. The electrode gaps between the emitting point and the grounded electrode were $100 \mathrm{~mm}$. The sharp pointed electrodes were connected to a power source that can supply alternating current (AC) high-voltage. In order to set the desired high-voltage parameters for HVAEF thawing, the power was connected to a voltage regulator, with an adjustable voltage ranging within $0-50 \mathrm{kV}$ for alternating current (AC) by a controller. The grounded plate electrode was an $80 \mathrm{~cm} \times 40 \mathrm{~cm}$ rectangular stainless steel plate. Temperature and relative humidity were both measured. The voltage and current of HVAEF system were measured by a voltmeter and an ampere meter, respectively. Figures 1(b) and 1(c) show arrangement diagram and schematic diagram of the needle electrodes, respectively. The needles were $20 \mathrm{~mm}$ long. The diameter of needles is $1 \mathrm{~mm}$. The distance between two needle electrodes was $40 \mathrm{~mm}$. The needle electrodes were arranged in multiple rows and lined up by stainless steel wire. The distance between two stainless steel wires was $40 \mathrm{~mm}$. All the samples were spread in a single layer on the grounded plate electrode at random. The center temperature of samples was measured by a temperature sensor.

2.2. Experimental Method. The soft tofu was procured from a local market near Inner Mongolia University of Technology, Hohhot, China. The fresh soft tofu was cut into cubes $(3.5 \mathrm{~cm}$ $\times 3.5 \mathrm{~cm} \times 3.5 \mathrm{~cm}$ ) using a knife and immediately frozen at $-18^{\circ} \mathrm{C}$ in a refrigerator. The frozen samples were stored at $-18^{\circ} \mathrm{C}$ until use.

The frozen tofu was thawed at room temperature $20 \pm 1^{\circ} \mathrm{C}$ under an electric field generated by a high-voltage of 4,8 , $12,16,20,24$, or $28 \mathrm{kV}$ for alternating current (AC). The control samples were placed on the same kind of stainless steel plate and subjected to HVAEF experimental apparatus in the treatment room $(0 \mathrm{kV})$. The thawing relative humidity was $30 \pm 5 \%$, and the ambient wind speed was $0 \mathrm{~m} / \mathrm{s}$. A temperature sensor was inserted into the geometric center of the frozen tofu sample and recorded at $5 \mathrm{~min}$ intervals during the thawing process. Thawing was continued until the geometric center of the frozen tofu sample temperature reached $10^{\circ} \mathrm{C}$. The time required to raise the temperature at the center of the frozen tofu cube from $-10^{\circ} \mathrm{C}$ to $10^{\circ} \mathrm{C}$ was determined as thawing time. Each experiment was repeated three times and averaged. The electric field strength is calculated from the following equation:

$$
E=\frac{V}{G},
$$

where $E$ is electric field strength, $V$ is the thawing voltage, and $G$ is the gap between the emitting point and the grounded electrode. The thawing rate (TR) of frozen tofu samples $(\mathrm{g} / \mathrm{s})$ was calculated using the following equation:

$$
\mathrm{TR}=\frac{W}{T},
$$

where $W$ is weight of frozen tofu and $T$ is the thawing time of frozen tofu.

2.3. Determination of Evaporation, Thawing, and Drip Losses. Evaporation loss (EL), thawing loss (TL), and drip loss (DL) were determined by weighing the frozen and thawed tofu samples before and after the removal of surface water according to the following equations [4]:

$$
\begin{aligned}
\operatorname{EL}(\%) & =\frac{\left(M_{0}-M_{\mathrm{T}}\right)}{M_{0}}, \\
\mathrm{TL}(\%) & =\frac{\left(M_{0}-M_{\mathrm{TT}}\right)}{M_{0}}, \\
\mathrm{DL} & =\mathrm{TL}-\mathrm{EL},
\end{aligned}
$$


TABLE 1: Mathematical models applied to the thawing time curve.

\begin{tabular}{lc}
\hline Model name & Model equation \\
\hline Power & $T=a E^{b}$ \\
Exponential & $T=a e^{b E}$ \\
Linear & $T=a+b E$ \\
Logarithmic & $T=a+b \ln (E)$ \\
Quadratic & $T=a+b E+c E^{2}$ \\
Inverse & $T=a+b / E$ \\
S & $T=e^{(a+b / E)}$ \\
\hline
\end{tabular}

$T$ is thawing time of frozen tofu in min, $E$ is electric field strength in $\mathrm{kV} / \mathrm{cm}$, and $a, b$, and $c$ are constants of mathematical models.

where $M_{0}, M_{\mathrm{T}}$, and $M_{\mathrm{TT}}$ are the weight of the frozen tofu, the thawed tofu before removing surface water, and the thawed tofu after surface water removal, respectively. Each experiment was repeated three times and averaged.

2.4. Specific Energy Consumption. The specific energy consumption (SEC) for the HVAEF system during thawing of tofu was measured by an ampere meter and a voltmeter, respectively. The specific energy consumption of HVAEF system during thawing of frozen tofu was calculated using the following equation:

$$
\mathrm{SEC}=\frac{U I t}{W_{d}},
$$

where $U, I$, and $t$ and $W_{d}$ are voltage of HVAEF system $(\mathrm{V})$, current of HVAEF system (A), thawing time (s), and the weight of frozen tofu $(\mathrm{kg})$, respectively.

2.5. Mathematical Model and Statistical Parameter. The experimental thawing curves were fitted to the seven different empirical models in Table 1. The model best suited for describing the thawing rate curve of frozen tofu was selected based on the values of the statistical parameters at 4, 8, 12, 16, 20,24 , and $28 \mathrm{kV}$ for AC electric field, respectively.

The root mean square error (ERMS), reduced mean square of the deviation $\left(\chi^{2}\right)$, and modeling efficiency (EF) were used as the primary criteria to select the equation that best accounts for the variation in the thawing curves of the thawed samples [11-13]. ERMS gives the deviation between the predicted and experimental values. $\chi^{2}$ was used to determine the goodness of the fit: the lower the values of $\chi^{2}$, the better the goodness of the fit. EF also gives the model predictive power in relation to the thawing behavior of the product, and its highest value is 1 . These statistical values were calculated using the following equation:

$$
\begin{aligned}
& \text { ERMS }=\sqrt{\frac{1}{N} \sum_{i=1}^{N}\left(T_{\text {pre }, i}-T_{\text {exp }, i}\right)^{2}}, \\
& \chi^{2}=\frac{\sum_{i=1}^{N}\left(T_{\text {pre }, i}-T_{\text {exp }, i}\right)^{2}}{N-n}, \\
& \mathrm{EF}=\frac{\sum_{i=1}^{N}\left(T_{\text {exp }, i}-T_{\text {exp }, \text { mean }}\right)^{2}-\sum_{i=1}^{N}\left(T_{\text {pre }, i}-T_{\text {exp }, i}\right)^{2}}{\sum_{i=1}^{N}\left(T_{\text {exp }, i}-T_{\text {exp }, \text { mean }}\right)^{2}},
\end{aligned}
$$

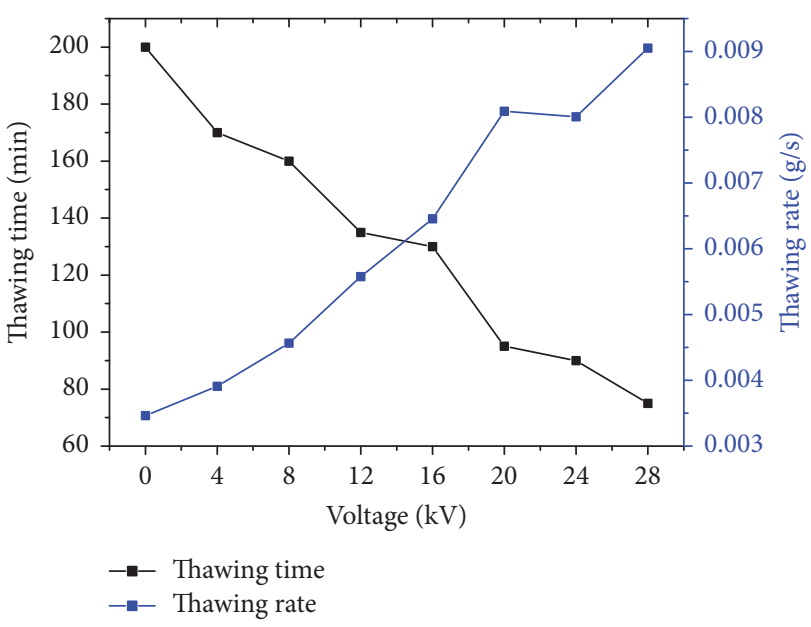

FIgURE 2: Thawing time and thawing rate of frozen tofu in different voltages.

where $T_{\exp , i}$ is the $i$ th experimental thawing time, $T_{\text {pre }, i}$ is the $i$ th predicted thawing time, $T_{\text {exp,mean }}$ is the mean value of experimental thawing time, $n$ is the number of constants in the thawing model, and $N$ is the number of observations.

2.6. Statistical Analysis. Single-factor analysis of variance was used to calculate the evaporation loss, thawing loss, and drip loss between the frozen tofu under alternating electric field and without electric field (control). The evaporation loss, thawing loss, and drip loss between different electric field were also calculated using single-factor analysis of variance. The differences in thawing time are considered statistically significant when $p<0.05$. The results reported in this study are presented as means \pm standard deviation (SD).

\section{Results and Discussion}

3.1. Thawing Time and Thawing Rate of Frozen Tofu by Different HVAEF Treatments. Figure 2 shows the effect of different voltages applied in the HVAEF on the thawing time and thawing rate. The frozen tofu was thawed at $20^{\circ} \mathrm{C}$ under applied voltages increasing from 4 to $28 \mathrm{kV}$ at increments of $4 \mathrm{kV}$ with a fixed electrode distance of $10 \mathrm{~cm}$. The thawing temperature of all samples was from $-10^{\circ} \mathrm{C}$ to $10^{\circ} \mathrm{C}$. The thawing times for voltages of $4,8,12,16,20,24$, and $28 \mathrm{kV}$ were $170,160,135,130,95,90$, and $75 \mathrm{~min}$, respectively, which were shortened significantly when compared with $200 \mathrm{~min}$ for the control $(0 \mathrm{kV})$. As voltage increased, the thawing time declined. The thawing rate of tofu samples treated with HVAEF increased compared to that of the control, and increasing the voltage had a major effect on the enhancement of the thawing rate, increasing by $0.1277,0.3180,0.6099$, $0.8638,1.3349,1.3116$, and 1.6119 times, respectively, at $4,8,12$, $16,20,24$, and $28 \mathrm{kV}$ voltages compared to that of the control $(0 \mathrm{kV})$. As can be seen, the results indicate that thawing rate increased with rise in voltage. These results agree with those studies which reported enhancement in thawing rate with increase of applied voltage $[3,4]$. 


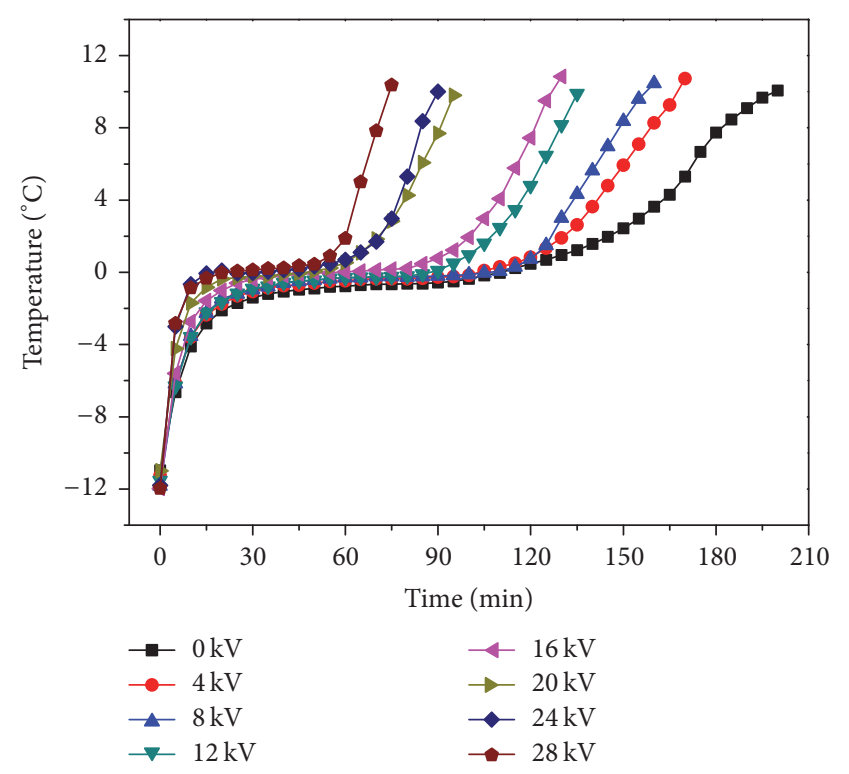

FIgURE 3: Changes in temperature during thawing of frozen tofu in different voltages.

Higher voltage or electric field strength can induce stronger ionic wind and higher wind velocity [14]. The enhancement in mass transfer rate could be attributed to the corona wind [15]. The corona wind produced impinges on the material and disturbs the liquid part of the thawing tofu, leading to thawing enhancement. The enhancement in thawing rate by the multiple points-to-plate could be attributed to electric wind created by each needle point electrode, resulting in a cumulative effect that could have greatly increased the thawing rate [16]. From Figure 2, it appears that the thawing rate is higher than the control when the voltage is lower than $15 \mathrm{kV}$. So, apart from corona wind under the AC electric field, another HVAEF thawing mechanism is possible because there is no corona wind. Specifically, as water molecules are highly polar they orient themselves in the direction of the electric field, which inturn would lead to the conversion of electrical energy into mechanical energy, thereby forcing water molecules out of the material [10]. This effect would be directly proportional to the electric field strength.

3.2. Center Temperatures of Frozen Tofu Thawed with Different HVAEF Treatments. Figure 3 illustrates the center temperatures of frozen tofu exposed to applied voltages $4,8,12,16,20,24,28 \mathrm{kV}$, and $0 \mathrm{kV}$ (control) at room temperature $\left(20^{\circ} \mathrm{C}\right)$. Center temperatures of frozen tofu were measured every $5 \mathrm{~min}$ and the initial thaw temperature of all samples was $-10^{\circ} \mathrm{C}$. The results indicate that the thaw temperatures increased rapidly, reaching about $-3^{\circ} \mathrm{C}$ in the first $10 \mathrm{~min}$. Within the last $20 \mathrm{~min}$, the thaw temperatures also increased rapidly, reaching about $10^{\circ} \mathrm{C}$. The thaw temperatures increased slowly between -2 and $0^{\circ} \mathrm{C}$. Most of the thawing time is consumed in raising the temperature from -2 to $0^{\circ} \mathrm{C}$. In the food freezing industry, $-5--1^{\circ} \mathrm{C}$ is often considered as the zone of maximum ice crystal
TABLE 2: The DL, EL, and TL of tofu under different voltages.

\begin{tabular}{lccc}
\hline Voltage $(\mathrm{kV})$ & DL $(\%)$ & EL $(\%)$ & TL $(\%)$ \\
\hline 0 (control) & $25.53 \pm 1.71^{\mathrm{a}}$ & $2.53 \pm 0.43^{\mathrm{a}}$ & $27.89 \pm 1.73^{\mathrm{a}}$ \\
4 & $23.81 \pm 0.81^{\mathrm{a}}$ & $2.60 \pm 0.22^{\mathrm{a}}$ & $26.42 \pm 1.02^{\mathrm{a}}$ \\
8 & $17.72 \pm 1.42^{\mathrm{b}}$ & $2.63 \pm 0.43^{\mathrm{a}}$ & $20.35 \pm 1.85^{\mathrm{b}}$ \\
12 & $11.70 \pm 1.19^{\mathrm{c}}$ & $2.91 \pm 0.29^{\mathrm{a}}$ & $14.60 \pm 1.18^{\mathrm{c}}$ \\
16 & $11.38 \pm 1.21^{\mathrm{c}}$ & $3.13 \pm 0.32^{\mathrm{a}}$ & $14.50 \pm 2.02^{\mathrm{c}}$ \\
20 & $9.49 \pm 1.88^{\mathrm{d}}$ & $5.25 \pm 0.48^{\mathrm{b}}$ & $14.74 \pm 2.07^{\mathrm{c}}$ \\
24 & $5.39 \pm 0.34^{\mathrm{e}}$ & $5.57 \pm 0.11^{\mathrm{b}}$ & $10.95 \pm 0.44^{\mathrm{d}}$ \\
28 & $3.44 \pm 0.16^{\mathrm{f}}$ & $5.03 \pm 0.12^{\mathrm{b}}$ & $8.48 \pm 0.28^{\mathrm{e}}$ \\
\hline
\end{tabular}

Data are shown as the mean \pm standard deviation (SD). For each treatment, means with different lower case letters are significantly different $(p<0.05)$.

formation $[2,5,7]$. Within this temperature range of slow thawing time $\left(-2-0^{\circ} \mathrm{C}\right)$, HVAEF treatment exerts its maximum effect. This result coincides with what has been found in other studies $[2,5]$.

3.3. EL, DL, and TL of Tofu. In this study, EL, DL, and TL were determined for the tofu samples with different voltages. Influence of voltage on the EL, DL, and TL of tofu is given in Table 2. When thawing loss is low, water holding capacity is high [2]. The results showed that evaporation loss increased with increasing applied voltage. The evaporation rate of material samples treated with HVAEF increased compared to that of the control, and increasing the voltage had a major effect on the enhancement of the evaporation rate [17-19]. Drip loss was less in the electric field treatments than in the control and decreased with increasing applied voltage. As can be seen, the results indicate that changes in voltage have significant effects on thawing loss. In other words, water holding capacity of tofu is improved using HVAEF thawing. This result does not coincide with what has been found in other studies $[2,4]$. The thawing process can cause the texture and the structural changes of the tofu [20]. Under same thawing method, there could have different results for the different materials. And the thawing loss is related to thawing time. The thawing time was shortened significantly under HVAEF compared to the control.

3.4. Effects of Voltage on Specific Energy Consumption. Figure 4 shows the specific energy consumption (SEC) of HVAEF system during thawing of tofu. The SEC of HVAEF system changes nonmonotonically with voltage, reaching a valley at $4-28 \mathrm{kV}$. He et al. found that the SEC of HVEF was significantly influenced by voltage and increased with increasing applied voltage $[3,5]$. The results showed that the electric field energy was not completely absorbed for thawing frozen tofu and a part of the energy absorbed by the control system. This will affect the energy efficiency of HVEF system.

3.5. Selection of the Best Mathematical Model. To obtain the superior mathematical models of frozen tofu, nonlinear regression analysis was carried on to estimate the constants and parameters of the seven thawing mathematical models given in Table 3 . The statistical results of the root mean 
TABLE 3: Statistical results, constants, and coefficients of mathematical models.

\begin{tabular}{|c|c|c|c|c|c|c|}
\hline Model name & $a$ & $b$ & $c$ & $\chi^{2}$ & ERMS & EF \\
\hline Power & 132.6482 & -0.3534 & & 260.3949 & 24.1728 & 0.8566 \\
\hline Exponential & 202.0317 & -0.3364 & & 60.0811 & 11.6113 & 0.9658 \\
\hline Linear & 188.5714 & -41.5179 & & 44.1071 & 9.9487 & 0.9722 \\
\hline Logarithmic & 137.3362 & -50.3776 & & 160.3174 & 18.9671 & 0.8991 \\
\hline Quadratic & 190.7143 & -45.0893 & 1.1607 & 54.5239 & 9.8935 & 0.9726 \\
\hline Inverse & 84.8487 & 40.2736 & & 470.6708 & 32.4990 & 0.6445 \\
\hline S & 4.5439 & 0.2641 & & 571.1514 & 35.8003 & 0.5686 \\
\hline
\end{tabular}

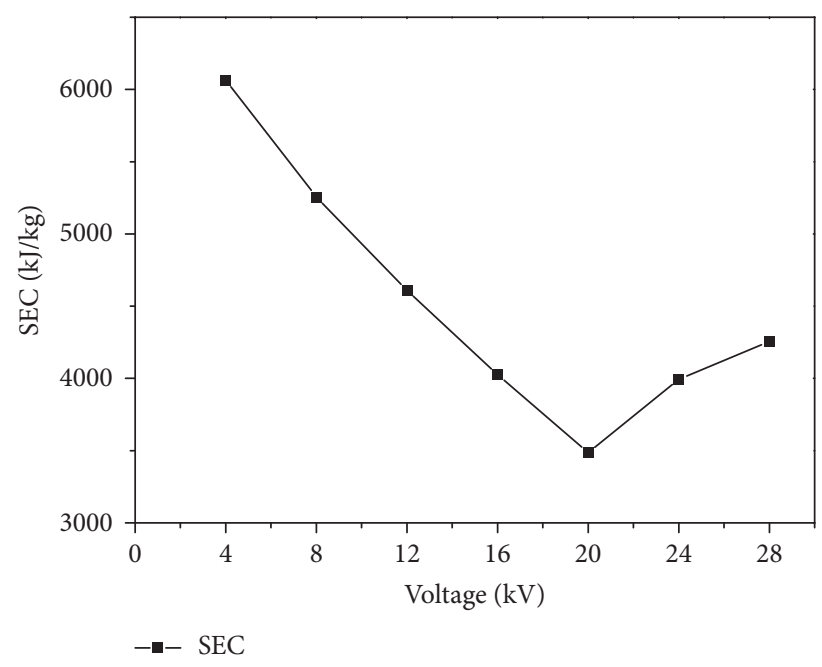

FIGURE 4: Effects of voltage on special energy consumption of tofu.

square error (ERMS), reduced mean square of the deviation $\left(\chi^{2}\right)$, and the modeling efficiency (EF) from mathematical models are given in Table 3. The results indicated that, in Exponential, Linear, and Quadratic models, the values of EF are above 0.96 , and $\chi^{2}$ and RMSE values ranged from 44.1071 to 60.0811 and 9.8935 to 11.6113 , respectively, indicating a good fitness. It is clear that all the three mathematical models could satisfactorily describe thawing time curves of frozen tofu treated by HVAEF. Of all the models fitted, the highest values of $\mathrm{EF}$ and the lowest values of $\chi^{2}$ could be obtained by the Quadratic model, but the lowest values of ERMS could be obtained by the Linear model. Thus, both the Linear and Quadratic models were selected as the best models to represent the thawing behavior of frozen tofu by HVAEF thawing technology. He et al. found that all the experimental results under high-voltage electric field fitted well with the Quadratic models for thawing time [3]; these results are in agreement with the present study.

\section{Conclusion}

The HVAEF technique can strengthen the thawing rate of frozen tofu. The thawing rate increases with strengthening applied voltage. By the root mean square error (ERMS), reduced mean square of the deviation $\left(\chi^{2}\right)$, and modeling efficiency (EF), both Linear and Quadratic models were found to be suitable for describing the thawing characteristics of frozen tofu under different voltages. Voltage has a major effect on thawing loss. Overall, HVAEF appears to be a feasible methodology for thawing frozen tofu. However, more studies are needed, especially for its comparison with other existing techniques for its full-scale utility.

\section{Conflicts of Interest}

All authors declare that they have no conflicts of interest regarding the publication of this paper.

\section{Acknowledgments}

This work was supported by National Natural Science Foundations of China (no. 51467015), College Students' Innovative and Entrepreneurial Training Program of Inner Mongolia (no. 201610128002), and Natural Science Foundation of Inner Mongolia Autonomous Region of China (no. 2017MS(LH)0507).

\section{References}

[1] M. Dalvi-Isfahan, N. Hamdami, A. Le-Bail, and E. Xanthakis, "The principles of high voltage electric field and its application in food processing: A review," Food Research International, vol. 89, no. 1, pp. 48-62, 2016.

[2] X. He, R. Liu, S. Nirasawa, D. Zheng, and H. Liu, "Effect of high voltage electrostatic field treatment on thawing characteristics and post-thawing quality of frozen pork tenderloin meat," Journal of Food Engineering, vol. 115, no. 2, pp. 245-250, 2013.

[3] X. He, R. Liu, E. Tatsumi, S. Nirasawa, and H. Liu, "Factors affecting the thawing characteristics and energy consumption of frozen pork tenderloin meat using high-voltage electrostatic field," Innovative Food Science and Emerging Technologies, vol. 22, pp. 110-115, 2014.

[4] A. Mousakhani-Ganjeh, N. Hamdami, and N. Soltanizadeh, "Impact of high voltage electric field thawing on the quality of frozen tuna fish (Thunnus albacares)," Journal of Food Engineering, vol. 156, pp. 39-44, 2015.

[5] A. Mousakhani-Ganjeh, N. Hamdami, and N. Soltanizadeh, "Thawing of frozen tuna fish (Thunnus albacares) using still air method combined with a high voltage electrostatic field," Journal of Food Engineering, vol. 169, pp. 149-154, 2016.

[6] A. Mousakhani-Ganjeh, N. Hamdami, and N. Soltanizadeh, "Effect of high voltage electrostatic field thawing on the lipid oxidation of frozen tuna fish (Thunnus albacares)," Innovative Food Science and Emerging Technologies, vol. 36, pp. 42-47, 2016. 
[7] C.-W. Hsieh, C.-H. Lai, W.-J. Ho, S.-C. Huang, and W.-C. Ko, "Effect of thawing and cold storage on frozen chicken thigh meat quality by high-voltage electrostatic field," Journal of Food Science, vol. 75, no. 4, pp. M193-M197, 2010.

[8] A. Wiktor, M. Schulz, E. Voigt, D. Witrowa-Rajchert, and D. Knorr, "The effect of pulsed electric field treatment on immersion freezing, thawing and selected properties of apple tissue," Journal of Food Engineering, vol. 146, pp. 8-16, 2015.

[9] X. He, G. Jia, E. Tatsumi, and H. Liu, "Effect of corona wind, current, electric field and energy consumption on the reduction of the thawing time during the high-voltage electrostaticfield (HVEF) treatment process," Innovative Food Science and Emerging Technologies, vol. 34, pp. 135-140, 2016.

[10] C. Ding, J. Lu, and Z. Song, "Electrohydrodynamic drying of carrot slices," PLoS ONE, vol. 10, no. 4, article e0124077, 2015.

[11] E. Meisami-asl, S. Rafiee, A. Keyhani, and A. Tabatabaeefar, "Mathematical modeling of moisture content of apple slices (Var. Golab) during drying," Pakistan Journal of Nutrition, vol. 8, no. 6, pp. 804-809, 2009.

[12] V. Demir, T. Gunhan, and A. K. Yagcioglu, "Mathematical modelling of convection drying of green table olives," Biosystems Engineering, vol. 98, no. 1, pp. 47-53, 2007.

[13] H. O. Menges and C. Ertekin, "Mathematical modeling of thin layer drying of golden apples," Journal of Food Engineering, vol. 77, no. 1, pp. 119-125, 2006.

[14] Y. Zhang, L. Liu, and J. Ouyang, "On the negative corona and ionic wind over water electrode surface," Journal of Electrostatics, vol. 72, no. 1, pp. 76-81, 2014.

[15] F. Hashinaga, T. R. Bajgai, S. Isobe, and N. N. Barthakur, "Electrohydrodynamic (EHD) drying of apple slices," Drying Technology, vol. 17, no. 3, pp. 479-495, 1999.

[16] Y. Bai, X. Li, Y. Sun, and H. Shi, "Thin layer electrohydrodynamic (EHD) drying and mathematical modeling of fish," International Journal of Applied Electromagnetics and Mechanics, vol. 36, no. 3, pp. 217-228, 2011.

[17] C. Ding, J. Lu, Z. Song, and S. Bao, "The drying efficiency of electrohydrodynamic (EHD) systems based on the drying characteristics of cooked beef and mathematical modeling," International Journal of Applied Electromagnetics and Mechanics, vol. 46, no. 3, pp. 455-461, 2014.

[18] A. Singh, V. Orsat, and V. Raghavan, "A comprehensive review on electrohydrodynamic drying and high-voltage electric field in the context of food and bioprocessing," Drying Technology, vol. 30, no. 16, pp. 1812-1820, 2012.

[19] S. Isobe, N. Barthakur, T. Yoshino, L. Okushima, and S. Sase, "Electrohydrodynamic drying characteristics of agar gel," Food Science and Technology Research, vol. 5, no. 2, pp. 132-136, 1999.

[20] Y. Xu, Y. Tao, and S. Shivkumar, "Effect of freeze-thaw treatment on the structure and texture of soft and firm tofu," Journal of Food Engineering, vol. 190, pp. 116-122, 2016. 

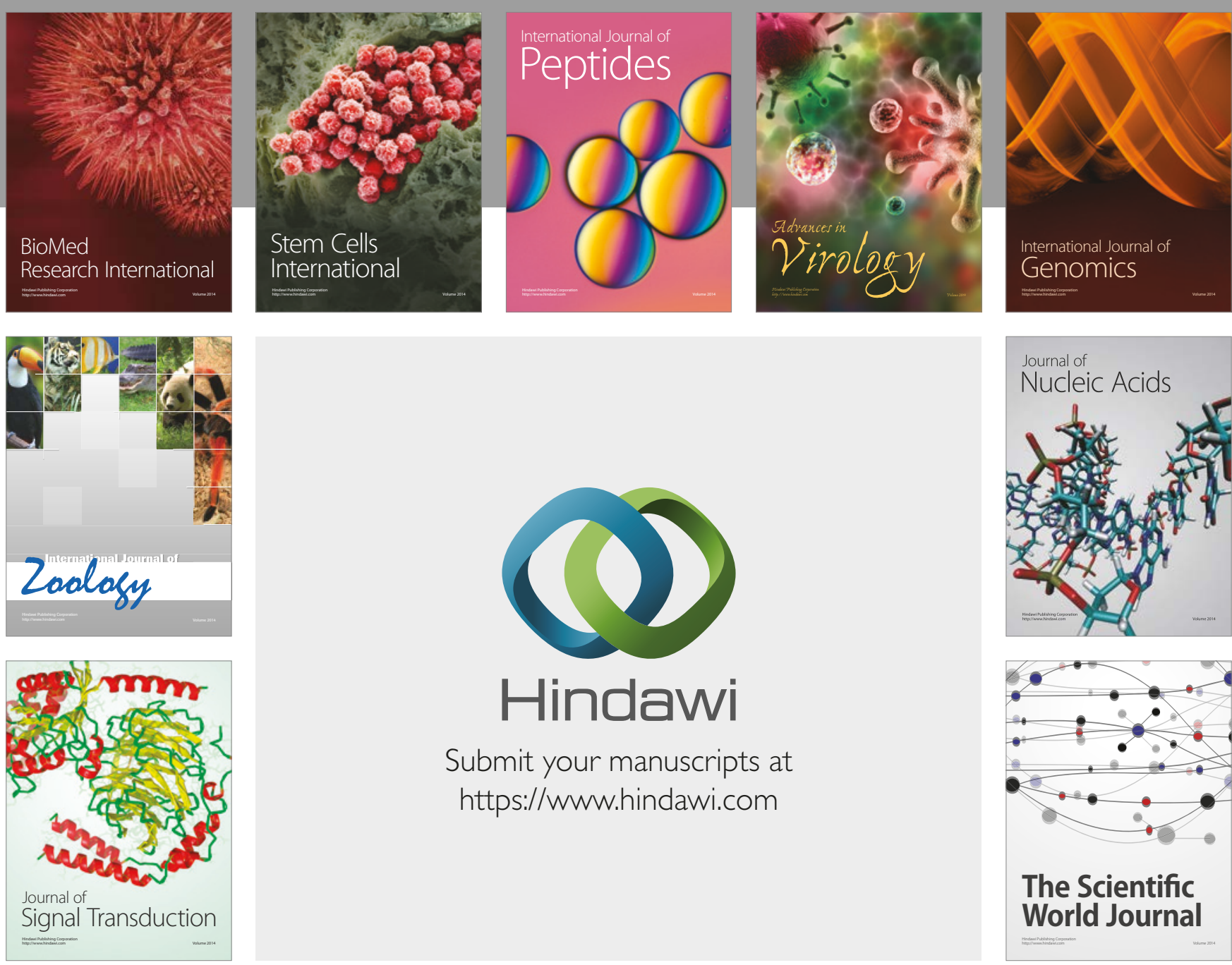

Submit your manuscripts at

https://www.hindawi.com
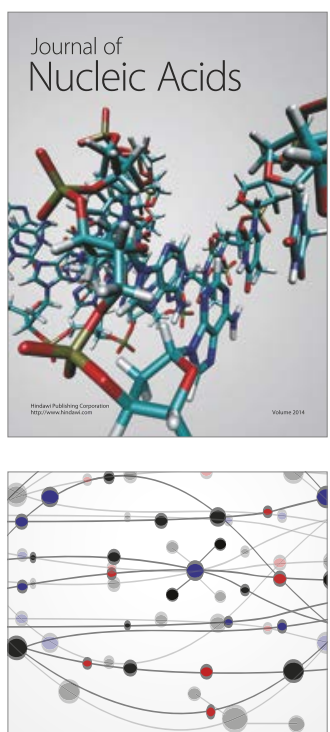

The Scientific World Journal

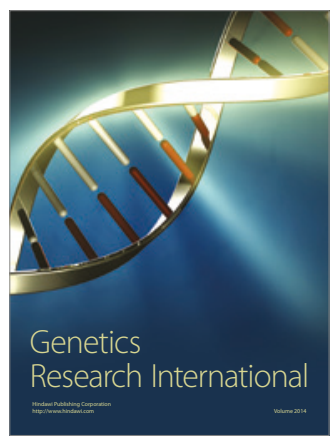

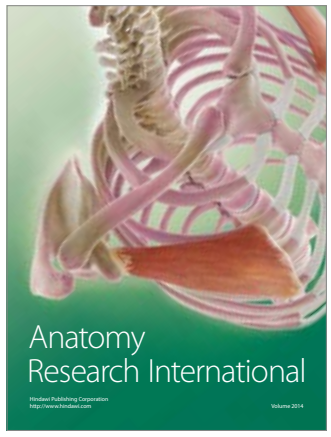

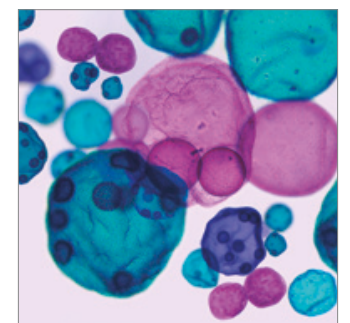

International Journal of Microbiology
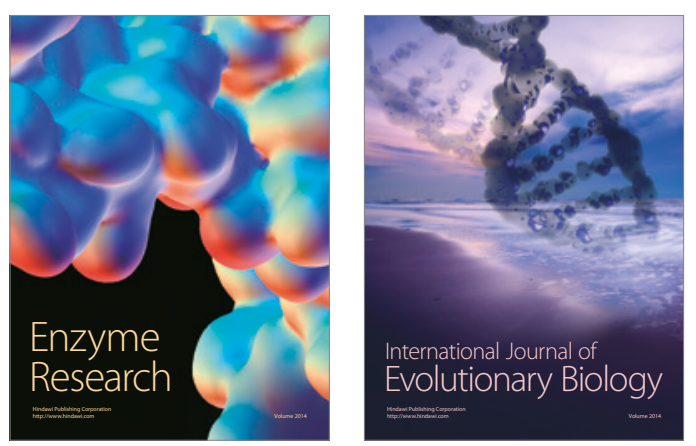
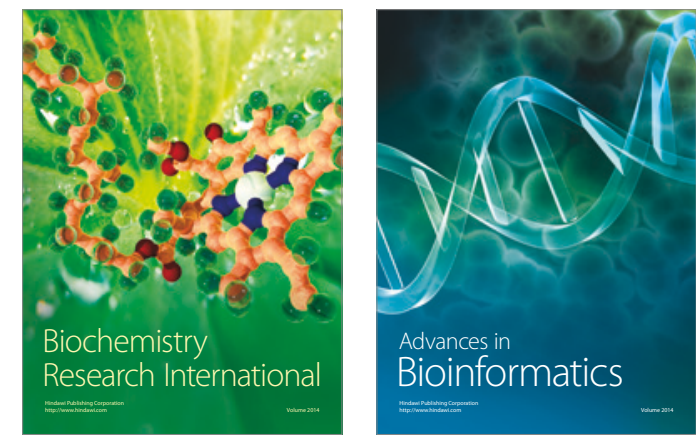

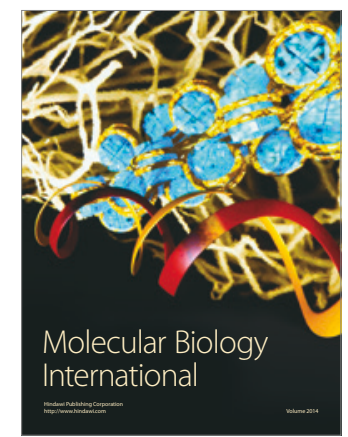

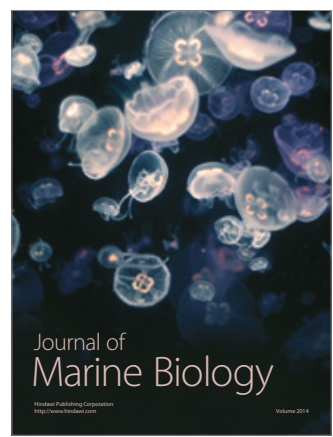

